\section{An interplanetary shock traced by planetary auroral storms from the Sun to Satum}

\section{Renée Prangé ${ }^{1}$, Laurent Pallier ${ }^{1}$, Kenneth C. Hansen ${ }^{2}$, Russ Howard ${ }^{3}$, Angelos Vourlidas ${ }^{3}$, Régis Courtin ${ }^{1} \&$ Chris Parkinson ${ }^{4}$}

${ }^{1}$ LESIA, Observatoire de Paris, 5 place Jules Janssen, 92195 Meudon, France ${ }^{2}$ Department of Atmospheric, Oceanic and Space Sciences, University of Michigan, Ann Arbor, Michigan 48109, USA

${ }^{3}$ Naval Research Laboratory, 4555 Overlook Avenue, SW Washington, DC 20375, USA

${ }^{4}$ Californian Institute of Technology, Jet Propulsion Laboratory and the NASA Astrobiology Institute, MS150-21 E. California Boulevard, Pasadena, California 91125, USA

A relationship between solar activity and aurorae on Earth was postulated $^{1,2}$ long before space probes directly detected plasma propagating outwards from the $\mathrm{Sun}^{3}$. Violent solar eruption events trigger interplanetary shocks ${ }^{4}$ that compress Earth's magnetosphere, leading to increased energetic particle precipitation into the ionosphere and subsequent auroral storms $s^{5,6}$. Monitoring shocks is now part of the 'Space Weather' forecast programme aimed at predicting solar-activity-related environmental hazards. The outer planets also experience aurorae, and here we report the discovery of a strong transient polar emission on Saturn, tentatively attributed to the passage of an interplanetary shock-and ultimately to a series of solar coronal mass ejection (CME) events. We could trace the shock-triggered events from Earth, where auroral storms were recorded, to Jupiter, where the auroral activity was strongly enhanced, and to Saturn, where it activated the unusual polar source. This establishes that shocks retain their properties and their ability to trigger planetary auroral activity thoughout the Solar System. Our results also reveal differences in the planetary auroral responses on the passing shock, especially in their latitudinal and local time dependences.

As for that of the Earth, outer-planet magnetospheres-the domain where the magnetic pressure of planetary origin exceeds the solar-wind kinetic (ram) pressure-are shaped by the solar-wind flow and they also display permanent aurorae near the magnetic poles at infrared, far-ultraviolet (FUV) and radio wavelengths ${ }^{7-11}$. Similarly, when a high-ram-pressure interplanetary shock wave reaches the planet, the magnetosphere is suddenly compressed. Among other consequences, the strength and distribution of currents and plasma are altered, and enhanced fluxes of energetic charged particles are expected to precipitate into the auroral ovals, which must be strongly activated. Indeed, strong statistical correlations were found between auroral radio emissions and the solarwind density and ram pressures ${ }^{12-16}$, particularly for the Earth and Saturn, whose magnetospheres are dominated by interactions with the solar wind at the polar-cap boundary ${ }^{17}$, between magnetic field lines open to the interplanetary magnetic field (IMF) and closed field lines. The correlation is looser at Jupiter, owing to the complex nature of its magnetosphere, partly controlled by processes of planetary origin ${ }^{18-20}$. For the Earth, an extended set of observations of the Sun, coordinated with measurements of the solar-wind physical parameters (density, velocity, composition, magnetic field strength and direction, and so on) and with various indicators of the auroral activity (among which are radio, visible and ultraviolet emissions), allows predictions of the ionosphere and atmosphere response to any interplanetary shock and or change in the direction of the IMF. This is not the case for the outer planets where there are no such coordinated observations, except during the Cassini fly-by of Jupiter in late 2000, where three occurrences of auroral radio storms coincided with the arrival of interplanetary shocks at the planet $^{21}$. In the absence of any image, the nature and location of the auroral events could not be compared with that of geomagnetic storms.

On 7 and 8 December 2000, FUV high-spatial-resolution images of Saturn were taken with the Hubble space telescope, HST (Fig. 1) with an excellent view of the southern polar region. The auroral oval morphology was very different from one day to the next. On 8 December, a narrow oval surrounding the pole at high latitude, and assigned to large-scale currrents at the boundary between open and closed field lines ${ }^{17}$, resembles the few Saturn aurorae observed earlier ${ }^{22}$; this configuration may be considered as a steady-state reference. By contrast, the aurora observed on the previous day is of

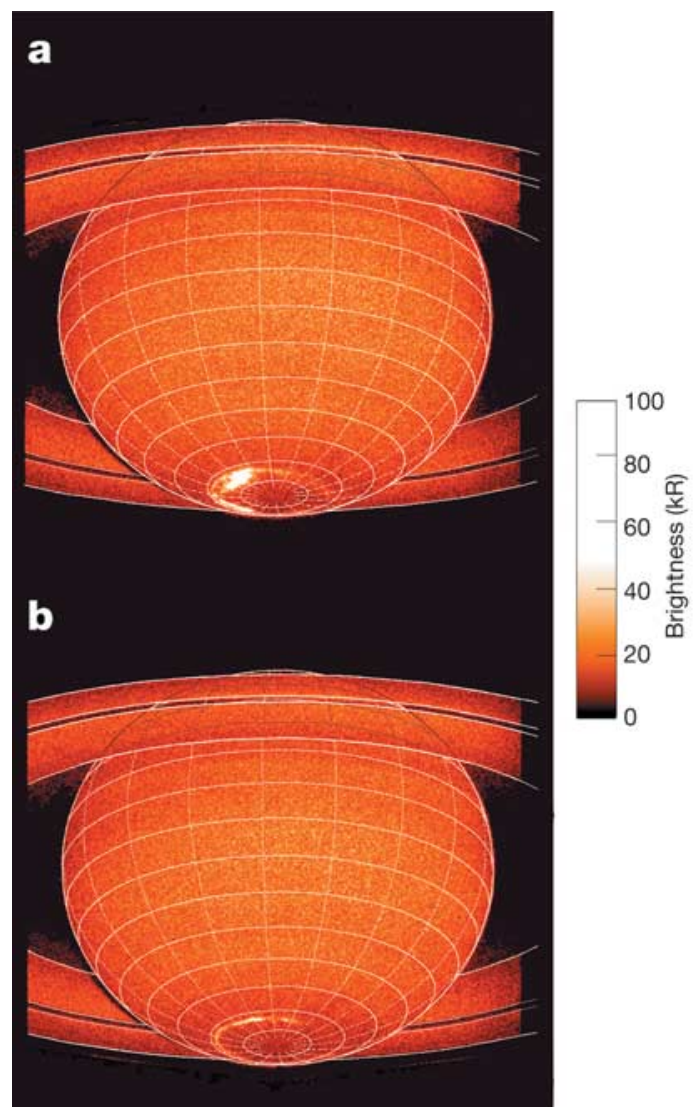

Figure 1 Discovery of an auroral storm on Saturn. The images, in a false-orange brightness colour code, were taken with the Space Telescope Imaging Spectrograph (STIS) onboard HST at 11:30 UT on 7 December 2000 (a), and 10:00 UT on 8 December (b). The exposure time is $480 \mathrm{~s}$, during which Saturn rotated by $\sim 5^{\circ}$. A spectral range including $\mathrm{H}_{2}$ Werner-Lyman auroral bands between $\sim 130$ and $\sim 165 \mathrm{~nm}$ is isolated by selecting the filter SRF2 in combination with the FUV MAMA solar blind detector. Nevertheless, some solar light is reflected by the planet disc in the FUV (up to $\sim 190 \mathrm{~nm}$ ). It is used to define the exact location of the planet on the detector and hence to correct pointing inaccuracies. After fitting an oblate spheroid plus ring system to the image, a grid of coordinates, with steps of $10^{\circ}$ in latitude and $20^{\circ}$ in longitude, is overlaid to determine the coordinates of the features of interest. The accuracy of the limb-fitting procedure is $\sim \pm 2$ pixels in each direction. The pixel size is $0.024 \times 0.024 \operatorname{arcsec}^{2}$ and the field of view is $24.7 \times 24.7 \operatorname{arcsec}^{2}$. The rotational phase of Saturn at the time of the observations is characterized by the longitude of the sub-Earth point, or local noon (SEP $=243^{\circ}$ and $283^{\circ}$ respectively in the images used here). The north polar region is hidden behind the ring system owing to the large tilt of Saturn's rotation axis at that time. Conversely, the whole region limited by the southern auroral oval is visible from Earth. Note the similarity in geometry and the differences in nightside brightness between the auroral oval on both days, but especially the very bright feature visible poleward of the dawnside oval on 7 December. This feature has totally disappeared on 8 December 
a kind never observed before: a similar oval is also present, but a very bright feature has transiently developed inside, on polar-cap open field lines. This suggests that we have witnessed for the first time an auroral storm at Saturn, which may be related to an as-yet-unknown interaction of the solar wind with Saturn's magnetosphere.

At that time, the Sun, the Earth, Jupiter and Saturn were nearly aligned (Fig. 2). Hence, any interplanetary shock would successively encounter the three planets along its radial propagation outward, even if its angular extent were small. A three-month observing campaign of Jupiter was also in progress, involving the Galileo spacecraft in the jovian magnetosphere, Cassini in the nearby solar wind, and the HST remotely imaging. On the other hand, observations of the Sun and of Earth's aurorae are performed on a continuous basis (for example, by the POLAR and IMAGE Earth orbiters), together with solar-wind measurements in the near vicinity of the Earth (for example, by the ACE and WIND spacecraft), to predict environmental hazards due to energetic particle precipitation into the auroral ionosphere (the 'Space Weather' programmes). A crude kinetic retracing of the solar-wind plasma suggests that if any shock had triggered the auroral storm on Saturn, it should have formed in early November near the Sun. Indeed, the LASCO coronographic spectrograph onboard the Solar and Heliographic Observatory (SOHO) detected a series of CMEs, five of them being directed toward the Earth over the 1-10 November period.

Thus all the conditions except one have been met that are required to investigate the interaction of an interplanetary shock with the Earth, Jupiter and Saturn consecutively. Only solar-wind measurements near Saturn were still needed to characterize the passage of a shock directly.

We could possibly have extrapolated them from the Cassini measurements near Jupiter, that is, $4 \mathrm{AU}$ upstream ( 1 astronomical unit, $\mathrm{AU}=149.5 \times 10^{6} \mathrm{~km}$ ) using a mere ballistic projection, $t_{1}=t_{0}+\Delta r / v_{\mathrm{sw}}$, and correcting for the difference in heliocentric longitude. However, this procedure, based on a purely 'kinematic' approach and ignoring plasma physics considerations, becomes more and more inaccurate as distance increases.

Therefore, we developed a more self-consistent method for obtaining the solar-wind conditions at the outer planets. We modelled the radial evolution of the plasma with a one-dimensional

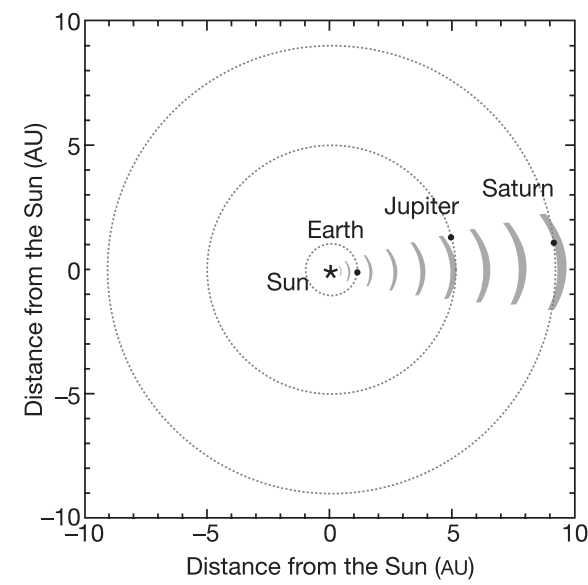

Figure 2 Geometrical configuration of the planets in late 2000. The relative heliocentric longitudes of the Earth, Jupiter and Saturn at the time when the interplanetary shock discussed in this study (in grey, schematic) passed each of them, are plotted. At the beginning (the end) of the passage, the angle from the Sun-Jupiter direction to the Sun-Earth direction is $23^{\circ}\left(17.5^{\circ}\right)$ as counted clockwise. For Saturn, these angles are $15^{\circ}$ and $9^{\circ}$, respectively. Consequently, the code used to propagate the solar wind is anticipated to be reasonably accurate at Jupiter, and more accurate at Saturn (see text). spherical magnetohydrodynamic code, using measurements made at Earth orbit as input. It was derived from the Versatile Advection $\mathrm{Code}^{23}$, which was modified to accept the ACE data as input at $1 \mathrm{AU}$, the inner boundary of the simulations. Although the assumption of spherical symmetry is not strictly valid, the comparison of the propagated solar wind with Cassini measurements shows a good match, particularly when the planet is close to alignment with the Sun-Earth direction ${ }^{24}$.

The 1-10 November series of CMEs triggered a series of five shocks, detected near the Earth (1 AU) by WIND and ACE about two days later. During this period, the POLAR Earth orbiter recorded correlated phases of geomagnetic storms and relaxation phases.

The code predicts that the individual shocks observed at $1 \mathrm{AU}$ later merged into a single long-duration disturbance (fast shocks can overtake slower ones). This disturbance is predicted to have passed the jovian magnetosphere in the 18-24 November timeframe, coinciding with the first of the jovian radio auroral storms mentioned above $e^{21}$. Compared with the Cassini in-situ data, the model shock arrives $\sim 0.7$ days too early. This small disagreement stems almost entirely from a combination of the IMF Parker spiral structure with the $\sim 20^{\circ}$ Earth-Jupiter difference in heliospheric longitude $e^{24}$. For Saturn, with a longitude difference of only $\sim 10^{\circ}$, this effect is expected to be smaller, and hence the predictions more accurate.

We used the code, validated at Jupiter, to propagate the shock further out, and we estimate that it passed Saturn between early 2 December and the morning of 8 December. Consequently, our first HST image exhibiting the polar storm was taken near the end of the shock interaction with Saturn's magnetosphere, while the second more 'typical' image is representative of a relaxation phase toward normal conditions, under a quiet ambient solar wind.

Thus we have assembled the first such synoptic view of the propagation of a CME-driven interplanetary shock from the Sun to Saturn, from in-situ measurements at the Earth and Jupiter (1 and $5 \mathrm{AU}$ ), and model-propagated plasma parameters at Jupiter and Saturn ( 5 and $9 \mathrm{AU}$ ). At each planet, a strong auroral response is recorded, based on POLAR images at the Earth, on Cassini radio observations at Jupiter, and on HST FUV images at Saturn. Figure 3 summarizes the observations. The solar-wind magnetic field strength and plasma ram pressure have been plotted in consecutive panels at the three planets. In parallel we display the last disturbed auroral image and the first 'relaxed' one for the Earth and Saturn. For Jupiter, we have instead plotted the radio output response.

We now compare the detailed characteristics of the auroral storms at the three planets, to progress towards an understanding of the physical processes at work during interactions between shocks and planetary magnetospheres.

At Earth, the quiet aurora, under solar-wind control, is located on the night side on closed magnetotail field lines. Compression of the magnetosphere by a shock leads to dramatic shifts towards low latitudes and brightenings of the auroral ovals (by up to three orders of magnitudes in extreme cases), which expand in local time both sides of midnight ${ }^{25}$ (see Fig. 3). Increased emission from the footprint of the dayside polar cusps, where 'direct' entry of solarwind plasma takes place, can also be observed as a result of reconnection events with the IMF and plasma injections ${ }^{26}$.

The Saturn 'normal' aurora also suggests some kind of solarwind-related local-time organization of the steady-state aurora. But in contrast with the Earth, the asymmetry in latitude and brightness develops between dawn and dusk ${ }^{17}$. During the storm, the auroral oval remains almost identical in size and location, but it is globally brighter by $50 \%$ (from $1.5 \times 10^{10}$ to $2.2 \times 10^{10}$ watts of total flux radiated in the $\mathrm{H}$ and $\mathrm{H}_{2}$ FUV spectrum). The brightness distribution along the oval also varies, its midnight-to-dawn sector (01:00-05:30 local time) becoming very active, up to $70 \mathrm{kR}$ ( $1 \mathrm{R}=10^{6}$ photons radiated in $4 \pi$ steradians), almost four times its next-day brightness. 

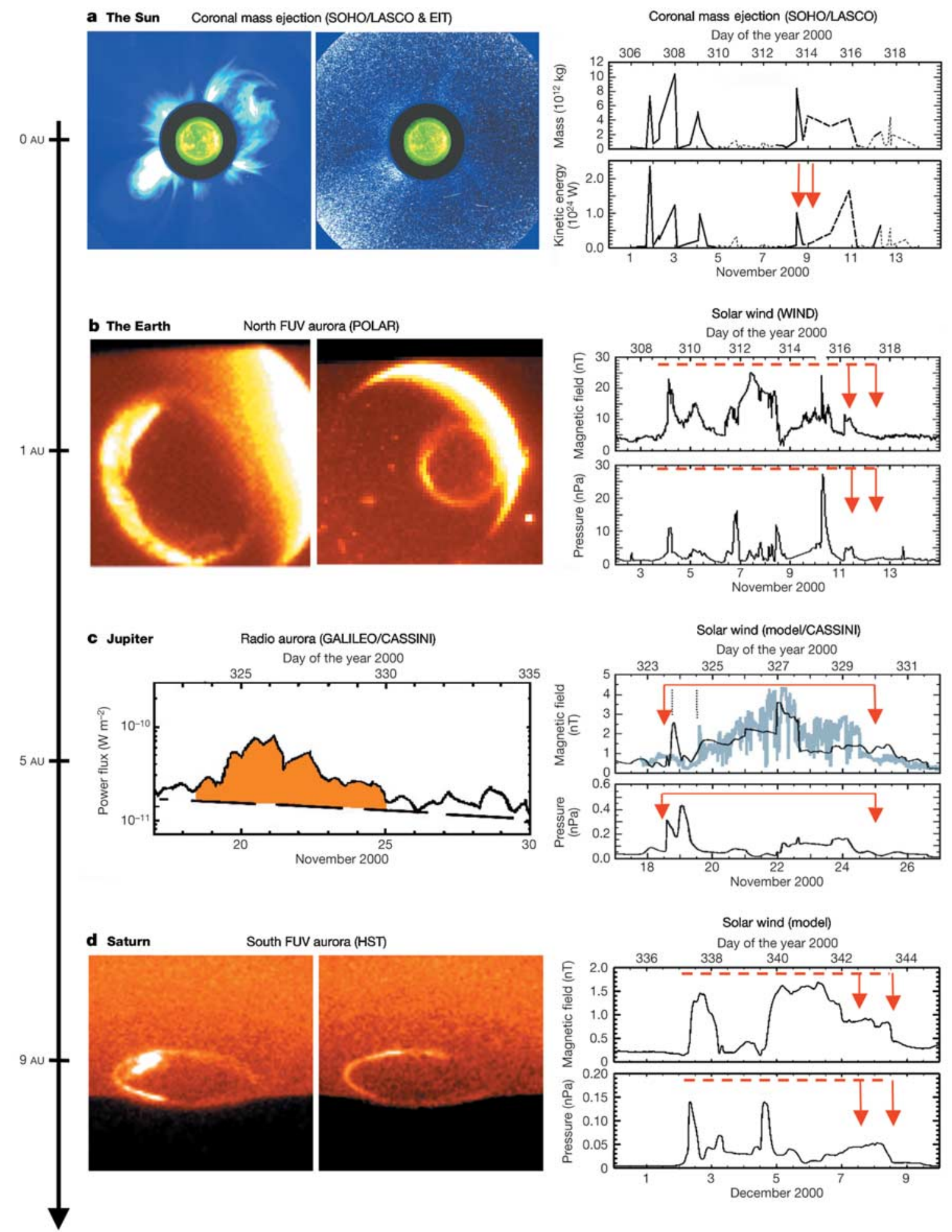

Figure 3 Synoptic view of the CME-driven shock propagation and auroral consequences. a, the Sun; b, the Earth; $\mathbf{c}$, Jupiter; $\mathbf{d}$, Saturn. Right column, solar-wind plasma parameters as a function of distance from the Sun ( $0 \mathrm{AU})$ to Saturn $(9 \mathrm{AU})$. For the CME, the mass $m$ and the kinetic energy $E_{c}=1 / 2 m v^{2}$ of the ejection event are plotted. For the interplanetary shocks, the magnetic field strength $B$, and the ram pressure $p=\rho v^{2}$. The dashed red lines and arrows indicate the duration of the event and the date of the images on the left. In a, thick solid lines, thick dashed lines, and thin dashed lines are for CMEs for which the geometry allows an encounter with the planets, marginally allows it, and does not allow it, respectively. In c, the magnetohydrodynamic-model propagated shock (solid line) is compared with the local Cassini measurements (thick grey line): the dotted vertical lines around 18 November highlight the small difference ( $\sim 0.7$ days) between the shock onset from the model and from the data. Left column, $\mathrm{SOHO}$ images of the solar CME (green brightness scale for EIT images of the solar disc, and blue code for LASCO coronographic images of the neighbouring solar wind) and of the planetary auroral responses to the shock (orange brightness scale). The Earth aurora has been recorded by the visible/FUV imager onboard POLAR (http://eiger. physics. uiowa.edu/ vis/images/). Because simultaneous auroral images are not available at Jupiter, we have displayed instead the global response at radio wavelengths ${ }^{19}$ 
In addition, there is a very bright, physically disconnected, feature inside the dawnside oval, at the footprint of polar-cap field lines. It extends polewards up to $\sim 78^{\circ} \mathrm{S}$ ( $\sim 6^{\circ}$ poleward of the oval), and is confined to the 5:30-11:00 local time sector. Its peak brightness (95 kR) largely exceeds that of the oval and its total auroral output, $2.4 \times 10^{10}$ watts, is comparable to the oval output despite its very limited spatial extent.

This suggests that, in response to the passage of the shock, the auroral oval mainly brightens at both planets on the night side, at the footprint of magnetotail magnetic field lines. However, Saturn does not exhibit the expansion towards lower latitudes that is typical of the geomagnetic storm oval. As for the bright polar-cap feature, it does not seem to have any terrestrial counterpart. It is reminiscent of the terrestrial polar cusp, frequently observed during dayside reconnection of closed field lines with the IMF, on or slightly poleward of the oval, and which is detected as a variable feature on Jupiter as well ${ }^{26}$. But, in contrast to the saturnian feature, the terrestrial polar cusp is close to noon ${ }^{27}$.

Recent studies, however, indicate that the IMF By east-west component (that is, dawn-dusk on Saturn) has a strong influence on the local-time position of the cusp and may shift it towards morning or afternoon by as much as several hours ${ }^{28,29}$. Even though polar cusps on Earth have not been observed as far as 6:00 local time, this is an issue that should be investigated. Unfortunately, the direction of the IMF cannot be extrapolated as the other solarwind properties can be. Another interpretation could be that strong reconnection at Saturn does not occur on the day side, but rather on the morning flank of the magnetopause, where it has been suggested that a Kelvin-Helmholtz instability triggers the saturnian auroral radio emissions ${ }^{30}$. It will be possible to check these hypotheses with local plasma measurements taken at Saturn by the Cassini mission.

Received 14 May; accepted 27 August 2004; doi:10.1038/nature02986.

1. de Mairan, J. J. Traité Physique et Historique de l'Aurore Boréale 245-279 (Mémoire de l'Académie Royale des Sciences, Imprimerie Royale, Paris, 1733).

2. Chapman, S. \& Ferraro, V. C. A. A new theory of magnetic storms. Nature 126, 129 (1930); Terr. Magn Atmos. Electr. 36, 171-186 (1931); Terr. Magn. Atmos. Electr. 37, 147-156 (1932).

3. Licht, A. L. Properties of the solar wind during sunspot minimum. J. Geophys. Res. 65, 1397-1400 (1960).

4. Greenstadt, E. W. Interplanetary magnetic effects of solar flares: Explorer 18 and Pioneer 5. J. Geophys. Res. 70, 5451-5452 (1965).

5. Snyder, C. W., Neugebauer, M. \& Rao, U. R. The solar wind velocity and its correlation with cosmicray variations and with solar and geomagnetic activity. J. Geophys. Res. 68, 6361-6370 (1963).

6. Axford, W. I. The interaction between the solar wind and the earth's magnetosphere. J. Geophys. Res. 67, 3791-3796 (1962)

Carr, T. D. Jupiter's decametric rotation period and the source A emission beam. Phys. Earth Planet. Inter. 6, 21-28 (1972)

8. Zarka, P. Auroral radio emissions at the outer planets: Observations and theories. J. Geophys. Res. 103, 20159-20194 (1998)

9. Broadfoot, A. L. et al. Extreme ultraviolet observations from Voyager 1 encounter with Jupiter. Science 204, 979-982 (1979)

10. Clarke, J. T. et al. Spatial imaging of hydrogen Lyman-alpha emission from Jupiter. Astrophys. J. 240, 696-701 (1980).

11. Broadfoot, A. L. et al. Extreme ultraviolet observations from Voyager 1 encounter with Saturn. Science 212, 206-211 (1981)

12. Gallagher, D. L. \& D'Angelo, N. Correlation between solar wind parameters and auroral kilometric radiation intensity. Geophys. Res. Lett. 8, 1087-1089 (1981).

13. Zarka, P. \& Genova, F. Low-frequency jovian emission and solar wind magnetic sector structure. Nature 306, 767-768 (1983)

14. Desch, M. D. \& Barrow, C. H. Direct evidence for solar wind control of Jupiter's hectometer wavelength radio emission. J. Geophys. Res. 89, 6819-6823 (1984).

15. Rabl, G. K. F. Periodicities of jovian broad-band kilometric radiation observed by Ulysses. Planet. Space Sci. 41, 1079-1083 (1993)

16. Desch, M. D. \& Rucker, H. O. The relationship between Saturn kilometric radiation and the solar wind. J. Geophys. Res. 88, 8999-9006 (1983).

17. Cowley, S. H. W., Bunce, E. J. \& Prangé, R. Saturn's polar ionospheric flows and their relation to the main auroral oval. Ann. Geophys. 22, 2685-2688 (2004).

18. Vasyliunas, V. M. in Physics of the Jovian Magnetosphere (ed. Dessler, A. J.) 395-453 (Cambridge Univ. Press, Cambridge, UK, 1990)

19. Prangé, R. et al. Detailed study of FUV Jovian auroral features with the post COSTAR Hubble Faint Object Camera. J. Geophys. Res. 103, 20195-20210 (1998).

20. Pallier, L. \& Prangé, R. More about the structure of the high latitude Jovian aurorae. Planet. Space Sci. 49, 1159-1173 (2001).

21. Gurnett, D. A. et al. Control of Jupiter's radio emission and aurorae by the solar wind. Nature 415 , 985-987 (2002).
22. Trauger, J. et al. Saturn's hydrogen aurora: Wide field and planetary camera 2 imaging from the Hubble Space Telescope. J. Geophys. Res. 103, 20237-20244 (1998)

23. Tòth, G. Versatile Advection Code; code available at 〈http://www.phys.uu.nl/ toth $\rangle$ (1994)

24. Hanlon, P. G. et al. On the evolution of the solar wind between 1 and $5 \mathrm{AU}$ at the time of the Cassin Jupiter flyby: Multispacecraft observations of interplanetary coronal mass ejections including the formation of a merged interaction region. J. Geophys. Res. 109, doi:10.1029/2003JA010112 (2004)

25. Frank, L. A. \& Craven, J. D. Imaging results from Dynamics Explorer. Rev. Geophys. 26, 249-283 (1988)

26. Pallier, L. \& Prangé, R. Detection of the southern counterpart of the jovian northern polar cusp. Shared properties. Geophys. Res. Lett. 31, doi:10.1029/2003GL018041 (2004).

27. Lockwood, M. et al. The ionospheric signature of flux transfer events and solar wind dynamic pressure changes. J. Geophys. Res. 95, 17113-17135 (1990).

28. Sandholt, P. E. Farrugia, C. J., Moen, J., Cowley, S. W. H. \& Lybekk, B. Dynamics of the aurora and associated currents during a cusp bifurcated event. Geophys. Res. Lett. 25, 4313-4316 (1981).

29. Milan, S. E., Lester, M., Cowley, S. W. H. \& Brittnacher, M. Dayside convection and auroral morphology during an interval of northward interplanetary magnetic field. Ann. Geophys. 18, 436-444 (2004).

30. Galopeau, P. H. M., Zarka, P. \& Le Quéau, D. Source location of Saturn's kilometric radiation: The Kelvin-Helmholtz instability hypothesis. J. Geophys. Res. 100, 26337-26410 (1998).

Acknowledgements This work is based partly on observations with the NASA/ESA HSTobtained at the STScI, which is operated by the AURA, Inc. for NASA.

Competing interests statement The authors declare that they have no competing financial interests.

Correspondence and requests for materials should be addressed to R.P. (renee.prange@obspm.fr).

\section{Optically programmable electron spin memory using semiconductor quantum dots}

\section{Miro Kroutvar, Yann Ducommun, Dominik Heiss, Max Bichler, Dieter Schuh, Gerhard Abstreiter \& Jonathan J. Finley}

Walter Schottky Institut, Technische Universität München, Am Coulombwall 3, D-85748 Garching, Germany

The spin of a single electron subject to a static magnetic field provides a natural two-level system that is suitable for use as a quantum bit, the fundamental logical unit in a quantum computer $^{1-3}$. Semiconductor quantum dots fabricated by strain driven self-assembly ${ }^{4}$ are particularly attractive for the realization of spin quantum bits, as they can be controllably positioned ${ }^{5}$, electronically coupled ${ }^{6}$ and embedded into active devices ${ }^{7-10}$. It has been predicted that the atomic-like electronic structure ${ }^{4}$ of such quantum dots suppresses coupling of the spin to the solidstate quantum dot environment ${ }^{11-14}$, thus protecting the 'spin' quantum information against decoherence ${ }^{15,16}$. Here we demonstrate a single electron spin memory device in which the electron spin can be programmed by frequency selective optical excitation. We use the device to prepare single electron spins in semiconductor quantum dots with a well defined orientation, and directly measure the intrinsic spin flip time and its dependence on magnetic field. A very long spin lifetime is obtained, with a lower limit of about 20 milliseconds at a magnetic field of 4 tesla and at 1 kelvin.

We begin by summarizing the operating principles of our optical spin storage device before presenting the measurements of the spin flip time, its dependence on magnetic field and the determination of the underlying mechanism. The structure of the devices investigated and the measurement techniques are summarized in Fig. 1. The samples consist of a single layer of self-assembled Ga(In)As quantum dots (QDs) embedded within the intrinsic region of a p-type GaAs Schottky photodiode ${ }^{17}$. Single electron-hole pair excitations (excitons) are generated directly in QD ground states by frequency- 